\title{
Pre-clinical alterations in cardiovascular phenotypes and their associations with metabolic profiles among obese youths
}

\author{
Elim Man*, Yiu-Fai Cheung, Pik-To Cheung \\ From 8th APPES Biennial Scientific Meeting \\ Darwin, Australia. 29 October - 1 November 2014
}

\begin{abstract}
Introduction
Childhood obesity and various obesity-related comorbidities have become a serious global healthcare problem. Predictive risk stratification and early detection of high-risk obese youths can potentially help modify the outcomes of obesity before irreversible damage taking place. Newer surrogate markers for assessing preclinical cardiovascular alterations have good predictive values for future cardiovascular risks. However, local data regarding obesity-related arterial alterations in youth are lacking at present.
\end{abstract}

\section{Aims}

To evaluate the 1) pre-clinical cardiovascular alterations; and 2) their associations with the metabolic profiles in obese Hong Kong Chinese youths.

\section{Study methods}

A total of 56 obese subjects and 58 healthy lean controls (M: F 1:1, age $17.0 \pm 2.06$ years) were recruited. Clinical, biochemical and arterial (structural and functional) parameters were obtained from all participants. Structural arterial parameters were determined by assessing carotid arterial intima-media thickness with ECG-synchronized ultrasound (Vivid 7, GE Medical Systems, Norway). Cross-sectional and regional arterial functions were determined by assessing the arterial stiffness $(\beta)$ of carotid arteries and pulse wave velocities of various arterial segments respectively with an automatic device (VP-2000; Colin Medical Technology, Japan). All ultrasonographic and arterial assessments were performed by a single researcher.

\footnotetext{
Department of Paediatrics \& Adolescent Medicine, Queen Mary Hospital, the
} University of Hong Kong, Hong Kong SAR, China

\section{Results}

Obese youths have worse preclinical arterial phenotypes both structurally and functionally, hence higher risks of developing cardiovascular disease in the future $(\mathrm{p}<0.0001)$. Important independent risk factors for preclinical arterial alterations in obese youths, including body mass indices (BMI), waist circumferences, blood pressures and plasma alanine aminotransferase (ALT) levels were demonstrated $(\mathrm{p}<0.0001)$. Obese youths having co-morbid metabolic disturbances were associated with worse preclinical arterial phenotypes. The worst cardiovascular phenotypes were shown in obese youths with the highest triglycerides to high-density lipoproteins (Tg-to-HDL) ratios despite most of them having normal serum triglyceride and HDL levels. Furthermore, obese subjects with higher paediatric NAFLD fibrosis index (PNFI) scores [1] have worse cross-sectional arterial stiffness.

\section{Conclusions}

Assessment of preclinical cardiovascular phenotypes in asymptomatic obese youths provides clinicians a window for early identification of those at higher risks of developing future cardiovascular events. Evaluation of lipid compositions and obesity-related liver alterations may potentially help further stratify obese youths into subgroups with different degrees of cardiovascular risks.

Published: 28 April 2015

\section{Reference}

1. Nobili V, Alisi A, Vania A, Tiribelli C, Pietrobattista A, Bedogni G, et al: The pediatric NAFLD fibrosis index: a predictor of liver fibrosis in children with non-alcoholic fatty liver disease. BMC Med 2009, 7:21.

doi:10.1186/1687-9856-2015-S1-O44

Cite this article as: Man et al:: Pre-clinical alterations in cardiovascular phenotypes and their associations with metabolic profiles among obese youths. International Journal of Pediatric Endocrinology 2015 2015(Suppl 1):044. 